\title{
On the Use of Rotating-Disk Geometry for Evaluating the Drag-Reducing Efficiency of Polymeric and Surfactant Additives
}

\author{
S. Jafargholinejad ${ }^{1 \dagger}$, A. Pishevar ${ }^{2}$ and K. Sadeghy ${ }^{3}$ \\ ${ }^{I}$ Malek-ashtar University of Technology, Isfahan, Iran \\ ${ }^{2}$ Department of Mechanical Engineering, Isfahan University of Technology, Isfahan, 84156-83111, Iran \\ ${ }^{3}$ University of Tehran, Tehran, Iran \\ $\dagger$ Corresponding Author Email: shapoor.jafargolinejad@gmail.com
}

(Received February 5, 2010; accepted May 27, 2010)

\begin{abstract}
In this study we will examine the applicability of the flow induced by a rotating disk in evaluating the performance of polymeric and surfactant additives in reducing skin friction drag and effect of viscosity on drag reduction capability of polymeric and surfactant solutions. It is shown that these additives can dramatically reduce friction drag provided that the flow is occurring under turbulent conditions while they have no effect on Taylor instabilities. Based on the experimental data, a drag reduction in the range of $10 \%$ can be achieved with the effect becoming more pronounced the higher the Reynolds number.
\end{abstract}

Keywords: Drag reduction, Taylor instabilities, Couette flow, Rotating disk, Polymeric and surfactant additives.

\section{NOMENCLATURE}

$\begin{array}{ll}\text { a } & \text { Disk Radius } \\ b & \text { Disk thickness } \\ \mathrm{c} & \text { Radial gap between disk and enclosure } \\ \mathrm{d} & \text { Radial gap between concentric cylinders } \\ \mathrm{DR} & \text { Drag reduction percentage } \\ R_{i} & \text { inner cylinder radius } \\ R e & \text { Reynolds number }\end{array}$

\section{INTRODUCTION}

Reducing skin friction is a need in many industries encompassing aviation and naval applications. This is because a drop in skin friction can decrease fuel consumption for a given range. Alternatively, for a given fuel supply it can increase the vessel range. For reasons like these studies related to drag reduction of surface and underwater vehicles has been one of the prime concerns of engineers and scientists alike.

Over the past decade, several studies have qualitatively shown that certain polymers and surfactants have the potential to be used as drag reducer in turbulent pipe flow (Toms 1948; Virk 1971). But, quantitatively, not much information is available in the literature to compare the drag-reducing effectiveness of different additives and/or the concentration at which their effect is peaked. To achieve this goal, one needs an accurate drag-measuring device capable of detecting any subtle

\author{
s Axial gap \\ Ta Taylor number \\ $\mathrm{T}_{\mathrm{BS}} \quad$ Measured torque on Aluminum Disk \\ $T_{D R S} \quad$ Measured torque at the presence of additive \\ $U_{i} \quad$ inner cylinder velocity \\ $v \quad$ kinematic viscosity \\ $\omega$ angular velocity
}

change in the drag experienced by a vessel at the presence of an additive.

Rotational flow between two concentric cylinders has successfully been used in the past for this purpose (Hall et al. 2000; Jones et al. 1969; Koeltzsch 2003 Taylor 1936; Zhang et al. 2002). Similarly, flow between a rotating disk and a fixed plate have also been used for this purpose (Choi et al. 1999,2000; Ge at al 2007; Hong et al. 2008; Kim et al. 2001; Sohn et al. 2001; Watanabe et al. 1998). In the present study, use will be made of the rotating disk geometry to assess the drag reducing capability of water soluble polymers and surfactants.

The idea is to inject dilute solutions of these additives into the viscous sublayer and see how it affects the torque needed to drive the disk at a constant angular speed. Any drop in this torque can be attributed to the effect of these additives on the wall shear stress. 


\section{Experimental Procedure}

\subsection{Test Fluids}

Polyethylene glycol 6000 (a synthetic polymer supplied by Merck Co.) was dissolved in two different solvents: i) distillated water, and ii) glycerol, at different concentrations. The solutions so obtained will be referred to as "PEG" samples in subsequent sections. As to the surfactant, use was made of a natural soap derived from animal fat which was dissolved in the same solvents as mentioned above. The solutions so obtained will be referred to as "S" samples. All samples were stored in a cold and dark place for 24 hours before being used in the experiments.

\subsection{Apparatus Description}

For determining the rheological properties of the test samples, the Brookfield LVDV2+ viscometer in the concentric cylinder mode was used in this work (Fig. 1). This viscometer was able to send physical data to a computer via its RS232 port. Physical data such as rotational speed of the spindle, torque, viscosity, shear stress, shear rate, temperature, and time could then be accessed and processed using DVpro software supplied with the instrument. The viscometer was equipped with a high precision torque sensor having a capacity of $673.7 \times 10^{-7} \mathrm{~N}$.m. With this viscometer, the accuracy of viscosity measurement was $\pm 0.1 \%$ of the full scale range with a repeatability of $\pm 0.2 \%$. The instrument included an adapter (see Fig. 2) for measuring viscosity of ultra low-viscosity flu-ids. The radial gap distance between the inner (rotating) cylinder and the outer (stationary) cylinder is about $1.235 \mathrm{~mm}$ in this particular configuration. This adapter also includes a water jacket with two holes for circulating cooling water and also for controlling the temperature. Cooling water was supplied by a temperature bath (Shell Lab Co.) shown in Fig. 3. The temperature ranged from ambient temperature up to a temperature of $80^{\circ} \mathrm{C}$.

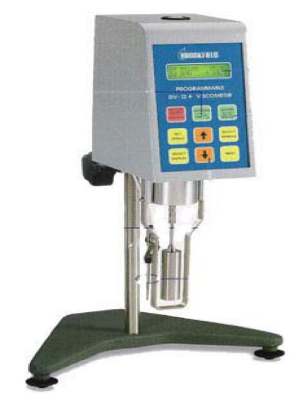

Fig.1. The Brookfield viscometer.
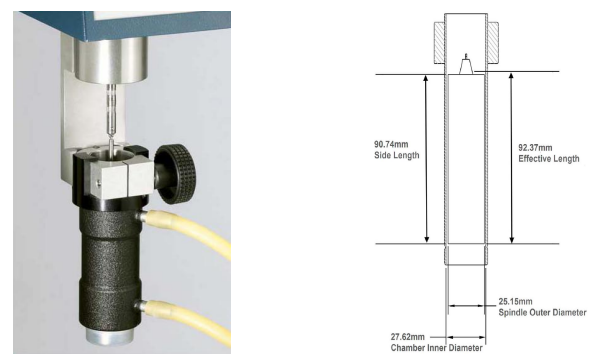

Fig. 2. Ultra low viscosity adapter with Dimension details

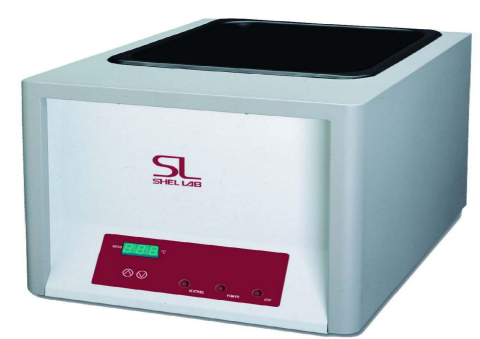

Fig. 3. Water bath

To evaluate the drag reduction efficiency of any given polymer or surfactant, a simple disk similar to that described in Ref. 8 was manufactured (see Fig.4). The disk was made of Aluminum and had a radius of $\mathrm{a}=7$ $\mathrm{cm}$ and a thickness of $\mathrm{b}=0.3 \mathrm{~cm}$. The enclosure in which the disk was rotating had a diameter of $18 \mathrm{~cm}$ and a height of $1.9 \mathrm{~cm}$. The disk could be rotated at a constant speed using the rotor system manufactured by Heidolph Company, Model RZR 2102 (Fig. 5). With this rotor system, the rotational speed of the disk could be controlled from 0 up to a maxi-mum of $2000 \mathrm{rpm}$. In practice, however, the device had to be run at rotational speed above $100 \mathrm{rpm}$ in order to have a measurable torque. The rotor system shown in Fig. 5 was equipped with a torque sensor having a capacity of 2 N.m with an accuracy of $0.001 \mathrm{~N}$.m. The device could display the resisting torque applied on the disk (at any given rpm) by the surrounding fluid.

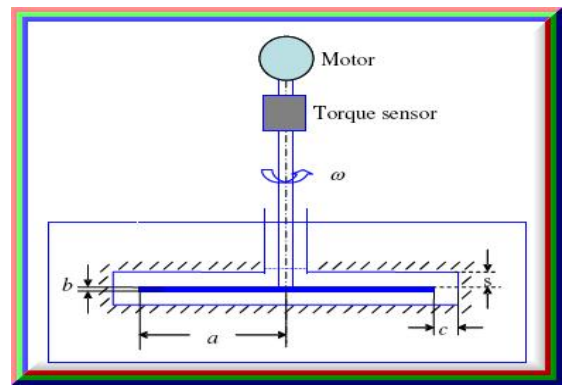

Fig. 4. Schematic of rotating disk apparatus

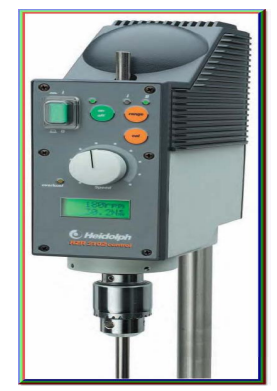

Fig. 5. Rotor apparatus for rotating the shaft

\subsection{Dimensionless Parameter Description}

The Taylor number is a dimensionless parameter that characterizes the importance of centrifugal force relative to the viscous force. There are two different definitions for the Taylor number in a Couette viscometer (White 2006; Schilichting 1979). The most common definition is: 
$\mathrm{Ta}=\frac{\mathrm{U}_{\mathrm{i}} \mathrm{d}}{v} \sqrt{\frac{\mathrm{d}}{\mathrm{R}_{\mathrm{i}}}}$

Where $U_{i}$ is the tangential velocity of the rotating inner cylinder, $\mathrm{d}$ is the radial gap distance, $v$ is the kinematic viscosity, and $R_{i}$ is the radius of the inner cylinder. Drag reduction efficiency of any additive can be judged by comparing the torque experienced by the disk while rotating in the solvent once without the additive and then with the additive at the same speed. It can be defined as:

$\% D R=\frac{T_{B S}-T_{D R S}}{T_{B S}} \times 100$

Where $\mathrm{T}_{\mathrm{BS}}$ and $\mathrm{T}_{\mathrm{DRS}}$ are the total torque (i.e., the torque exerted on both sides of the disk) in the base solvent and in the solution including the additive. The Reynolds number of the disk-induced flow is defined as:

$$
\operatorname{Re}_{\text {Disk }}=\frac{a^{2} \omega}{v}
$$

Where "a" is the disk radius, $\omega$ is the angular velocity, and $v$ is the kinematic viscosity. For the flow induced in the fluid by an enclosed rotating disk, the transitional $\mathrm{Re}$ is known to be around $3 \times 10^{5}$ (White 2006). Using this critical Reynolds number as the criterion to ensure that the flow is indeed turbulent, we have reached to the conclusion that the disk should be rotated at rotational speeds above $580 \mathrm{rpm}$ to guarantee that flow is truly turbulent.

The coefficient of torque for rotating disk geometry is defined as:

$$
C_{M}=\frac{M}{\frac{1}{2} \rho \omega^{2} a^{5}}
$$

Where $\mathrm{M}$ is Torque measured by sensor and $\rho$ is fluid density.

\section{RESULTS AND DISCUSSION}

Figures 6 and 7 show the effect of the polymer and surfactant additives on the viscosity data of the base solvent (a $60 \%-40 \%$ combination of water and glycerol) at a typical temperature of $28^{\circ} \mathrm{C}$. It is to be noted that the data presented in these two figures were obtained using the Brookfield viscometer in the concentric cylinder mode with the inner cylinder rotating and the outer cylinder fixed. As can be seen in these two figures, the absolute viscosity of mentioned of "PEG" and "S" solutions is $2.95 \mathrm{mPa} . \mathrm{s}$ and $2.92 \mathrm{mPa} . \mathrm{s}$ respectively. As expected, the viscosity of the solvent (i.e., water) is increased in the presence of the additives. A comparison between Figs. 6 and 7 reveals that the viscosity of polymer-extended solution is higher than surfactant-extended solution over the range of the shear rates tested. It is also interesting to note that at shear rates above roughly $100 \mathrm{~s}^{-1}$, viscosity of the solutions is increased by an increase in the shear rate. Since "PEG" and "S" solutions are known to be shear-thinning, this apparent shear-thickening should be regarded as an artifact of the instrument. That is, concentric cylinder viscometers are known to be vulnerable to Taylor instability when the inner cylinder is rotating with the outer cylinder being fixed. This instability exhibits itself as a secondary flow, the so-called Taylor vortices. In practice, these vortices significantly increase the resisting torque thus giving rise to erroneous reading for the viscometer. This phenomenon is observed when the outer cylinder is stationary and the inner cylinder is rotating. For Newtonian fluids, it exhibits itself when the Taylor number exceeds a critical value of 41.3. It is one of the main objectives of the present work to see how the occurrence of this phenomenon is affected by the polymer and surfactant additives.

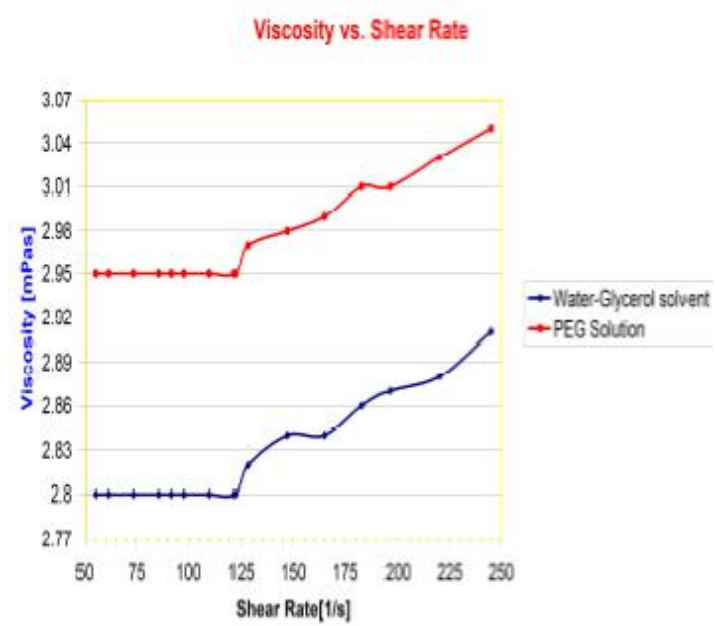

Fig. 6. Viscosity data for the test polymer solutions.

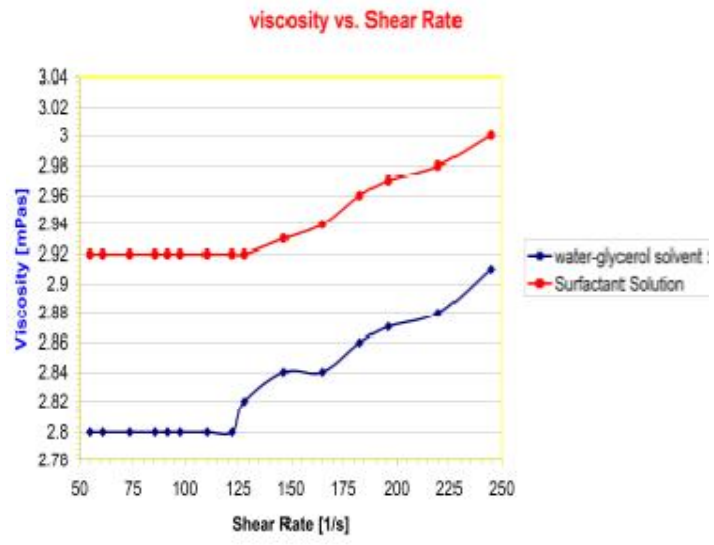

Fig. 7. Viscosity data for the test surfactant solutions.

Figure 8 shows the relative increase in the resisting torque as a function of the Taylor number for distilled water with/without the two additives mentioned previously. As can be seen, the rise in torque is increased by an increase in the Taylor number (see Taylor in-stability region in the Fig. 8). This means that neither the polymer nor the surfactant is capable of inhibiting Taylor instabilities, although there appears to be a small shift in the critical Taylor number (43 instead of 41.3 for Newtonian fluids). We also tried other concentration of the two additives and reached to the conclusion that although these additives can not significantly delay Taylor instability, they reduce its intensity as rise in torque is lowered as compared with the distillated water values. 


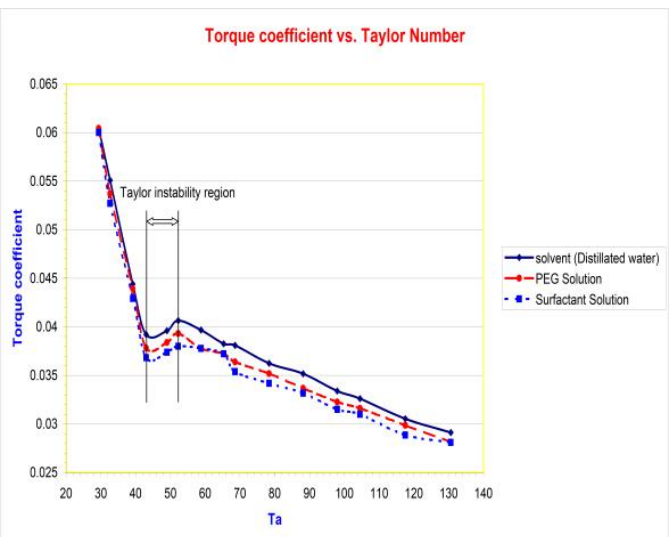

Fig. 8. Coefficient of torque as a function of the Taylor number

We are now at a stage to present our experimental data for the drag-reducing efficiency of our polymer and surfactant additives. Figure 9 shows the torque applied on the rotating disk as a function of the rotational speed. As can be seen in this figure, the resistive torque is reduced at the presence of these additives.

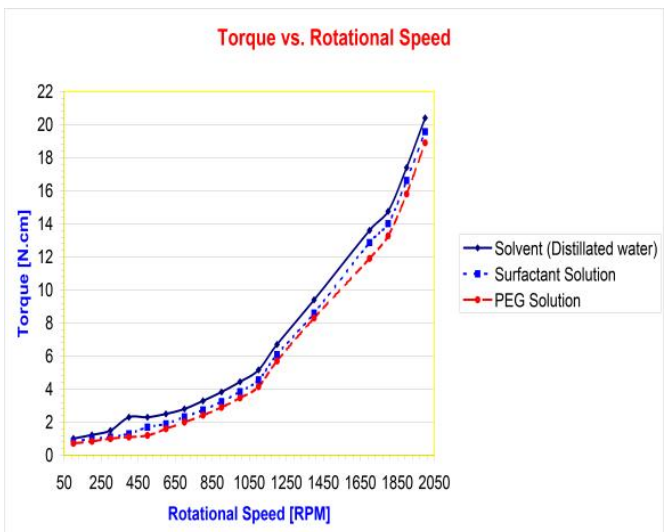

Fig. 9. Torque exerted on the shaft of the rotating disk at different rotational speeds.

The percentage of the drag reduction versus the Reynolds number is presented in Fig.10. As can be seen in this figure, the percentage of drag reduction is reduced by an increase in the Reynolds number. This can be attributed to the fact that polymer additives will lose their efficiency as a drag reducer if they undergo mechanical degradation at high shear rates (corresponding to high Reynolds numbers). Similarly, for surfactants a breakdown of micelles and/or network structures at high shear rates may be responsible for the drop in their efficiency as a drag reducer.

It is also interesting to note that in spite of the higher viscosity of the polymer solution with respect to surfactant solution, its drag reduction efficiency is better than the surfactant solution (at least, over the range of the Reynolds number used in this work). In addition, in spite of the higher viscosity of polymer and surfactant solutions, as compared with the solvent (i.e., distilled water), one could say that the drag reducing mechanism of these additives is perhaps due to their effect on the structure of the boundary layer near the wall.

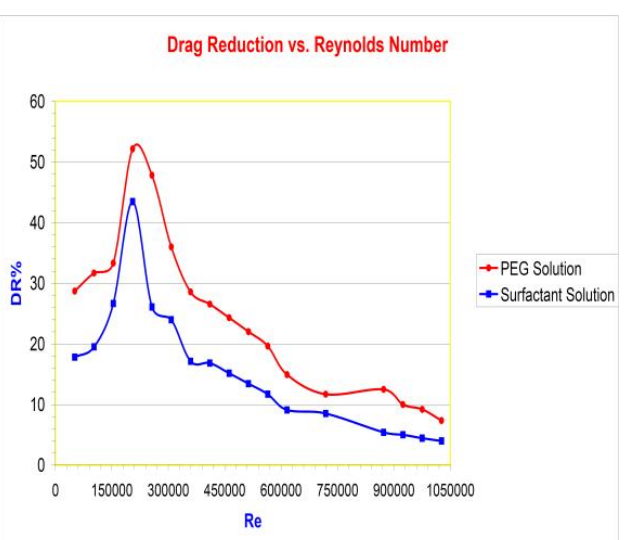

Fig. 10. Drag reduction percentage as a function of the Reynolds number.

\section{CONClusion}

Based on the results obtained in the present work it can be concluded that polymers and surfactants can reduce skin friction under turbulent flow condition. This is quite interesting realizing the fact that the viscosity of the solvent itself is increased at the presence of these additives. It appears that these additives affect flow kinematics in such a way that the velocity gradients adjacent to the wall are affected to such an extent that the overall wall shear stress is reduced.

\section{REFERENCES}

Choi, H.J., C.A. Kim, and M.S. Jhon (1999). Universal drag reduction characteristics of polyisobutylene in a rotating disk apparatus. Polymer 40, 4527-4530.

Choi, H.J., C.A. Kim, J.H. Sung, C.B. Kim, W. Chun, and M.S. Jhon (2000). Universal drag reduction characteristics of saline water-soluble poly (ethylene oxide) in a rotating disk apparatus. Colloid Polym Sci 278, 701-705.

Choi, K.S., K. Prasad, and T.V. Truong (1996). Emerging Techniques in Drag Reduction. European Drag Reduction Meeting, Berlin.

Ge, W., Y. Zhang, and J.L. Zakin (2007). Surfactant turbulent drag reduction in an enclosed rotating disk Apparatus. Exp Fluids 42, 459-469.

Gerhart, P., R. Gross, and J. Hochstein (1992). Fluid Mechanics. Addison Wesley, New York.

Hall, T., and D. Joseph (2000). Rotating cylinder drag balance with application to riblet. Experiments in Fluids 29, 215-227.

Hong, C.H., H.J. Choi, and J.H. Kim (2008). Rotating disk apparatus for polymer-induced turbulent drag reduction. J. of Mechanical Science and Technology, 22, 1908-1913.

Hough, G.R. (1992). Viscous Flow Drag Reduction. AIAA: Progress in Astronautics and Aeronautics 72, ISBN: 0915928442. 
S. Jafargholinejad et al. / JAFM, Vol. 4, No. 2, Issue 1, pp. 1-5, 2011.

Jones, W.M. and D.E. Marshall (1969). Relaxation effects in Couette flow between rotating cylinders. Brit. J. Appl. Phys. 2(6), 809.

Koeltzsch, K., Y. Qi, R.S. Brodkey,and J.L. Zakin (2003). Drag reduction using surfactants in a rotating cylinder geometry. Experiments in Fluids 34, 515-530.

Kim, C.A., D.S. Jo, H.J. Choi, C.B. Kim, M.S. Jhon (2001). A High-precision Rotating Disk Apparatus for Drag Reduction Characterization. ELSEVIER Polymer Testing 20, 43-48.

Sohn, J.I., C.A. Kim, H.J. Choi, and M.S. Jhon (2001). Drag-reduction effectiveness of xanthan gum in a rotating disk apparatus. Carbohydrate Polymers 45, 61-68.

Schlichting, H. (1979). Boundary Layer Theory. Seventh Edition, McGraw-Hill Series in Mechanical Eng.

Sellin, R.H. (1989). Drag Reduction in Fluid Flows: Techniques for friction control. 4th Int. Conf. on Drag Reduction, Switzerland, Halsted Press.

Steven, L., D.R. Ceccio, M.P. Dowling, and M. Solomon (2007). Influence of Surface Roughness on Polymer Drag Reduction. Technical report, Contract No. R00110610057.

Toms B.A. (1948), In Proc. of 1st Int. Congress on Rheology, Amsterdam:North Holland Pub. Co, 135.

Taylor, G.I. (1936). Fluid Friction between rotating cylinders I-Torque Measurements. Proc Roy Soc A, 223, 546-564.

Virk, P.S. (1971). Drag reduction in rough pipes. $J$. Fluid Mech. 45(2), 225-246.

Watanabe, K., and S. Ogata (1998). Drag Reduction for a Rotating Disk with Highly Water-Repellent Wall. JSME Int. Journal. Series B 41(3), 683-775.

White, F.M. (2006). Viscous Fluid Flow. Third Edition, McGraw-Hill Series in Mechanical Eng.

Wells, C.S. (1969). Viscous Drag Reduction. Plenum Press, New York, ISBN: 77-76496.

Zhang, X., J. Tian, L. Wang, and Z. Zhou (2002).Wettability effect of coatings on drag reduction and paraffin deposition prevention in oil. Journal of Petroleum Science and Engineering 36, 87-95. 